\title{
Radial Evolution of Stochastic Heating in Low- $\beta$ Solar Wind
}

\author{
Mihailo M. Martinovic ${ }^{1,2,3}$ (1) , Kristopher G. Klein ${ }^{1}$ (1) , and Sofiane Bourouaine ${ }^{4}$ (1) \\ ${ }^{1}$ Lunar and Planetary Laboratory, University of Arizona, Tucson, AZ 85719, USA; mmartinovic@email.arizona.edu \\ ${ }^{2}$ LESIA, Observatoire de Paris, Meudon, France \\ ${ }^{3}$ Department of Astronomy, Faculty of Mathematics, University of Belgrade, Serbia \\ ${ }^{4}$ Florida Institute of Technology, Melbourne, FL, USA \\ Received 2019 March 14; revised 2019 May 20; accepted 2019 May 21; published 2019 July 2
}

\begin{abstract}
We consider the radial evolution of perpendicular ion heating due to the violation of magnetic moment conservation caused by Alfvén and kinetic Alfvén wave turbulence. This process, referred to as stochastic heating $(\mathrm{SH})$, can be quantified by the ratio between the average velocity fluctuations at the ion gyroradius and the perpendicular ion thermal speed $\epsilon \equiv \delta v / v_{t \perp}$. Using $17 \mathrm{yr}$ of Helios observations, we constrain how much energy could be dissipated by this mechanism between 0.29 and 0.98 au. We find that $\mathrm{SH}$ likely operates throughout the entire inner heliosphere, but that its radial dependence is steeper than that of empirically derived dissipation rates, with $r^{-2.5}$ being compared with $r^{-2.08}$. This difference is significantly increased in fast solar wind streams to $r^{-3.1}$ compared with $r^{-1.8}$
\end{abstract}

Key words: plasmas - solar wind - turbulence

\section{Introduction}

Over the last six decades, numerous hydrodynamic models of the solar wind (Parker 1958; Sturrock \& Hartle 1966; Wolff et al. 1971), which rely on thermal conduction as the driver of the solar wind heating and acceleration, were developed. These models are unable to explain the existence of fast solar wind streams and large proton temperatures measured at 1 au (see e.g., Echim et al. 2011 and references therein), suggesting that there are additional heating mechanisms operating above the coronal base. Among other candidate processes (Markovskii et al. 2010; Drake \& Swisdak 2012; Osman et al. 2012; Kasper et al. 2013; Vech et al. 2018), Alfvén wave (AW) turbulent dissipation has been widely examined (Coleman 1968; Hollweg 1973; Villante 1980; Velli et al. 1989; Matthaeus et al. 1999) as one of the possible answers to this open question, ever since large amplitude AWs were first observed in the interplanetary medium (Belcher et al. 1969; Belcher \& Davis 1971).

In this work we focus on heating of the solar wind due to turbulent Alfvénic fluctuations with perpendicular scales of the order of the proton gyroradius $\left(k_{\perp} \rho \sim 1\right.$, where $k_{\perp}$ is the component of the wavevector $\boldsymbol{k}$ perpendicular to the magnetic field $\boldsymbol{B})$. At these scales, the turbulent Alfvénic cascade transforms into a kinetic Alfvén wave (KAW) cascade (see, e.g., Bale et al. 2005; Howes et al. 2008b). As the turbulent power is wavevector anisotropic with $k_{\perp} \gg k_{\|}$(Goldreich \& Sridhar 1995; Cho \& Lazarian 2004; Schekochihin et al. 2009; Chen et al. 2010; Mallet et al. 2015), the KAWs have frequencies smaller than the proton cyclotron frequency $\Omega_{p}$ (Howes et al. 2008a), making the collisionless damping due to cyclotron resonances negligible (Lehe et al. 2009). Also, for the magnetically dominated, low- $\beta$ environment expected close to the Sun, the ion thermal speed $v_{t}$ is much smaller than the Alfvén speed $v_{\mathrm{A}}$, quenching Landau or transit time damping, which requires $\omega \approx k_{\|} v_{t \|}$ (Quataert 1998; Hollweg 1999), where $v_{t \|}$ is the parallel thermal speed. While dissipation via Landau damping has been identified in magnetosheath turbulence (Chen et al. 2019) and gyrokinetic numerical simulations of turbulence (Klein et al. 2017) using the fieldparticle correlation technique (Klein \& Howes 2016; Howes et al. 2017), such linear mechanisms are not accessible for the measured particle and wave parameters at proton gyroradius scales in the inner heliosphere. This difficulty motivated numerous authors (Dmitruk et al. 2002, 2004; Hollweg \& Isenberg 2002; Drake et al. 2009; Lehe et al. 2009) to investigate perpendicular ion heating due to nonlinear processes.

Here, we examine the effects of stochastic heating ( $\mathrm{SH}$ ) by low frequency turbulent Alfvénic fluctuations near the proton gyroradius scale. This mechanism is based on the idea that magnetic moment $\mu_{m}=m_{p} v_{\perp}^{2} / 2 B$ invariance, $m_{p}$ being the proton mass, is violated in a turbulent plasma if the amplitude of the velocity fluctuations is sufficiently large, leading to heating of the protons. In the theoretical work of Chandran et al. (2010), it was shown that this process can be phenomenologically quantified by the parameter

$$
\epsilon=\frac{\delta v}{v_{t \perp}}
$$

where $\delta v$ is the rms amplitude of velocity fluctuations at the proton gyroradius scale, $v_{t \perp}=\sqrt{2 k_{\mathrm{b}} T_{\perp} / m_{p}}$ is the perpendicular thermal speed, and $k_{\mathrm{b}}$ is the Boltzmann constant. As $\epsilon$ increases, the particle orbits become increasingly chaotic and depart from their smooth gyromotion, leading to perpendicular diffusion. Given a particular amplitude of turbulent fluctuations $\delta v$, and assuming $\beta_{\|} \lesssim 1$, the associated heating rate due to $\mathrm{SH}$ is given by

$$
Q_{\perp}=\frac{c_{1}(\delta v)^{3}}{\rho_{p}} \exp \left[-\frac{c_{2}}{\epsilon}\right],
$$

where $c_{1}$ and $c_{2}$ are dimensionless parameters of order unity that can be extracted from numerical simulations. Chandran et al. (2010) found that for test-particle simulations with $\epsilon_{\text {crit }}=0.19, Q_{\perp}$ equalled half of the turbulent cascade power at $k_{\perp} \rho \sim 1$.

Observational tests for the presence of $\mathrm{SH}$ were performed by Bourouaine \& Chandran (2013), who calculated $\epsilon$ for low- $\beta$, fast solar wind using Helios data at three different radial 
distances $(0.29,0.4$, and $0.64 \mathrm{au})$. At all three distances, similar values of $\epsilon \approx 0.048$ were found. The authors concluded that, for values of $c_{1}$ and $c_{2}$ consistent with RMHD turbulence simulations, $Q_{\perp}$ is comparable to an empirically derived heating rate. A more recent statistical study performed by Vech et al. (2017) using Wind data at 1 au reported that the proton temperature anisotropy $T_{\perp} / T_{\|}$, likely the result of some preferential perpendicular heating mechanism such as $\mathrm{SH}$, and the scalar proton temperature $T_{p}$ linearly increased with $\epsilon \geqslant \epsilon_{\text {crit }} \approx 0.025$, a turbulence amplitude for which $\mathrm{SH}$ is expected to be negligible. These results present a dilemma; does SH continuously operate throughout the entire inner heliosphere, or are observations at 1 au a remnant of processes that happened days ago, when the solar wind was closer to the Sun?

Pursuing an answer to this question, we use a method analogous to the one described in Bourouaine \& Chandran (2013) to process $17 \mathrm{yr}$ of observations from both Helios 1 and 2 (Porsche 1981a, 1981b) and investigate possible dependencies of $\epsilon$ and $Q_{\perp}$ as a function of the radial distance from the Sun. An overview of the method is given in Section 2. Processing of the E2 magnetometer data required removing the effects of instrument noise and saturation, as well as the spacecraft spin, as explained in Section 3. Section 4 contains the results of the statistical study, which we compare with theoretical (Chandran et al. 2011) and empirical (Hellinger et al. 2011, 2013) models in order to examine the importance of $\mathrm{SH}$ at different radial distances, as well as inferences for enhanced heating at distances closer than the Helios perihelia as predicted by Kasper et al. (2017). Discussion and possible implications of our results, as well as their limitations, are discussed in Section 5.

\section{Method}

Our method of data processing is based on that described in Bourouaine \& Chandran (2013). We use the Helios E1 instrument (Schwenn et al. 1975) proton corefit data set with $\sim 40$ s time resolution provided by Stansby et al. (2018) to obtain 10 minute averages of parallel and perpendicular proton temperatures, $T_{\perp}$ and $T_{\|}$and mean angle $\theta$ between the solar wind flow and the magnetic field $\boldsymbol{B}$. The proton density $n_{p}$, density of $\alpha$ particles $n_{\alpha}$, and solar wind bulk velocity $v_{\mathrm{sw}}$ were obtained from the data set on NASA CDAWeb. ${ }^{5}$ In periods when data were missing from the CDAweb data set, we used corefit values. We note that the corefit data set only considers the core component of the proton distribution for densities, bulk velocities, and temperatures; proton density from the corefit data set is shown to be about $80 \%$ of the total proton density, while $v_{\mathrm{sw}}$ is on average $\sim 1.5 \%$ lower. For these intervals, we used an averaged value of $n_{\alpha} \approx 0.04 n_{p}$ (Matthias et al. 2001). This approach enables us to work with $48 \%$ more measurements $(259,801$ instead of 175,834 , see Figure 1(a)), while increasing the uncertainty in the results by up to $10 \%$, less than the uncertainties that emerge from E2 instrument data processing and model parameters (Sections 3 and 4). High resolution magnetic field data from the E2 magnetometer (Musmann et al. 1975) are provided by the instrument team. ${ }^{6}$ From this data set, we calculate the parallel plasma $\beta$, proton

\footnotetext{
5 https://cdaweb.gsfc.nasa.gov/index.html HELIOS1_40SEC_MAG-PLASMA, HELIOS2_40SEC_MAG-PLASMA

6 http://helios-data.ssl.berkeley.edu/
}

gyroradius and proton gyrofrequency, defined as

$$
\begin{gathered}
\beta_{\|}=\frac{2 \mu_{0} n_{p} k_{\mathrm{b}} T_{\|}}{B^{2}} \\
\rho_{p}=\frac{v_{t \perp}}{\Omega_{p}} \\
\Omega_{p}=\frac{e_{c} B}{m_{p}}
\end{gathered}
$$

where constants $\mu_{0}$ and $e_{c}$ stand for the magnetic permeability of vacuum and the unity charge, respectively.

Given that beam-like components of the proton velocity distribution appear mostly in the direction parallel to $\boldsymbol{B}$ (Marsch et al. 1982), we assume the $T_{\perp}$ extracted from the corefit data set is a good measure of the total perpendicular temperature. On the other hand, the scalar temperature reported by the corefit and core-and-beam data sets can vary significantly, with their ratios varying from 0.5 to 2 , where the corefit temperature is $\sim 5 \%$ lower on average (Stansby et al. 2018). Consequently, our results for $T_{\|}$may be slightly underestimated, lowering the accuracy of our low- $\beta_{\|}$criteria given in Equation (3), but not to a degree that should affect the results presented in this work.

In order to derive values for $\epsilon$ and $Q_{\perp}$, we must determine the amplitude of the velocity fluctuations at the proton gyroscale, $\delta v$. As measurements of the proton velocity by Helios are not sufficiently fast to capture gyroscale fluctuations, we assume the fluctuations are Alfvénic and write

$$
\delta v=\frac{\sigma v_{\mathrm{A}} \delta B}{B}
$$

where $\sigma=1.19$ is a dimensionless constant used by Chandran et al. (2010) for a spectrum of randomly phased KAWs with $k_{\perp} \rho_{\perp} \sim 1$ and the Alfvén velocity is given by

$$
v_{\mathrm{A}}=\frac{B}{\sqrt{\mu_{0}\left(n_{p}+4 n_{\alpha}\right) m_{p}}} .
$$

The magnetic field fluctuations at the proton gyroscale $\delta B$ are defined as

$$
\delta B=\left[\frac{\pi}{C_{0}\left(n_{s 12}\right)} \int_{e^{-0.5 f_{p}}}^{e^{0.5 f_{p}}} P_{B}(f) d f\right]^{1 / 2},
$$

where $f_{p}=v_{\mathrm{sw}} \sin \theta / \rho_{p}$ is the convected gyrofrequency, $P_{B}(f)$ is the magnetic field power spectra,

$$
\begin{gathered}
C_{0}\left(n_{s 12}\right) \\
=\frac{C_{0}\left(n_{s 1}\right) \int_{e^{-0.5 f_{p}}}^{f_{b}} P_{B}(f) d f+C_{0}\left(n_{s 2}\right) \int_{f_{b}}^{e^{0.5 f_{p}}} P_{B}(f) d f}{\int_{e^{-0.5 f_{p}}}^{f_{b}} P_{B}(f) d f+\int_{f_{b}}^{e^{0.5 f_{p}}} P_{B}(f) d f} \\
C_{0}\left(n_{s}\right)=\int_{0}^{\pi / 2} \cos \phi^{n_{s}-1} d \phi=\frac{\pi^{0.5} \Gamma\left[\frac{n_{s}}{2}\right]}{2 \Gamma\left[\frac{n_{s}+1}{2}\right]}
\end{gathered}
$$




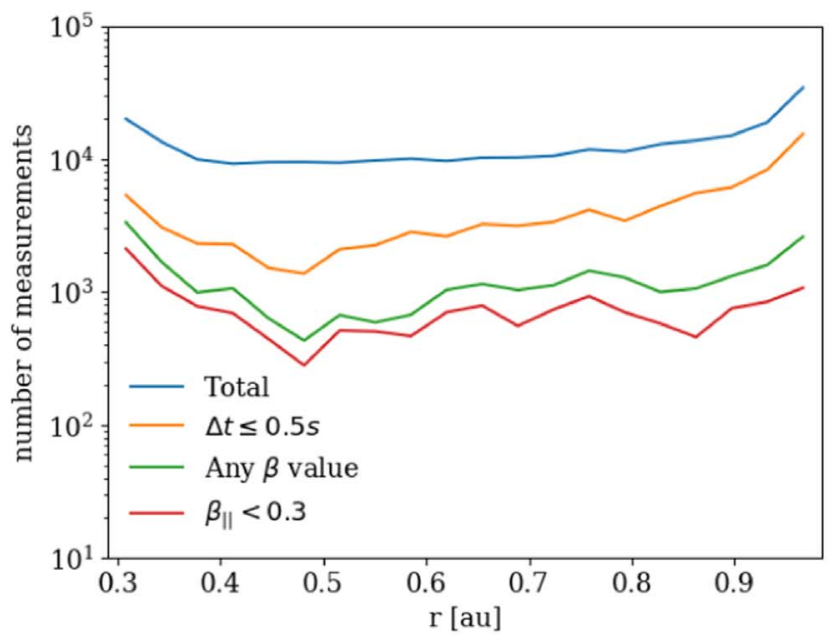

(a)

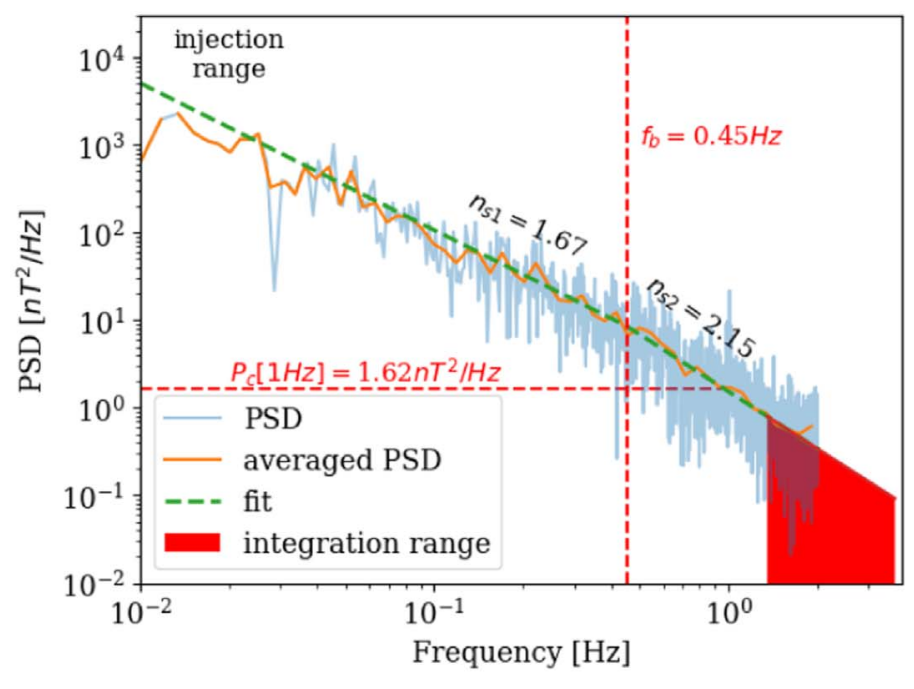

(b)

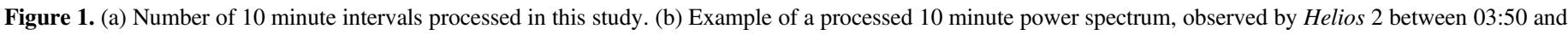
04:00 on 1976 April 14.

are geometric terms described in Bourouaine \& Chandran (2013) and Vech et al. (2017); $f_{b}$ is the break frequency between the inertial and dissipation ranges. ${ }^{7}$

To evaluate Equation (8), we process the E2 magnetic field power spectra $P_{B}(f)$ in the same way as was done by Bourouaine \& Chandran (2013), applying the algorithm illustrated in Figure 1(b). First, we calculate a standard trace power spectrum as a sum of fast Fourier transforms of each of the three magnetic field components (blue solid line). The instrument sampling rate is not constant, but rather divided into regimes with timesteps $\Delta t \approx n 0.25 \mathrm{~s}$, with $n$ a positive integer. For this analysis we make use only of periods where the resolution was $\Delta t \approx 0.25 \mathrm{~s}$ or $\approx 0.5 \mathrm{~s}$, for a Nyquist frequency to 2 or $1 \mathrm{~Hz}$, respectively. The peak at $f=1 \mathrm{~Hz}$ due to the spacecraft spin is removed by a notch filter. The frequency range is then divided into logarithmically spaced regions and averaged within each of these regions (orange solid line).

From the processed spectrum, we look for the break point frequency $f_{b}$ in the logarithmic slope that is characteristic of the transition between magnetohydrodynamic (MHD) and ion scales in a similar fashion as was done by Vech et al. (2017; see Figure 1(a) of that article). Throughout each spectrum, we perform a series of linear fits for logarithmic windows that contain $10 \%$ of the data points. We define the starting frequency of the fit with the largest slope, $n_{s 2}$, to be $f_{b}$. We then perform a new linear fit for $f<f_{b}$ to find logarithmic slope $n_{s 1}$ for the inertial range. These fits provide the power spectral density (PSD) $B^{2} / \nu$ at $1 \mathrm{~Hz}, P_{c 1}$ and $P_{c 2}$, for the portions of the spectra below and above $f_{b}$, respectively. Due to the possibility that $f_{b}$ is larger than the Nyquist frequency or is covered by instrument noise, we model $P_{B}$ as the piecewise function

$$
\begin{aligned}
& P_{B}(f)=10^{P_{c 1}} f^{-n_{s 1}}, f<f_{b} \\
& P_{B}(f)=10^{P_{c 2}} f^{-n_{s 2}}, f>f_{b}
\end{aligned}
$$

\footnotetext{
7 Equation (8) is similar to Equation (2) from Vech et al. (2017), but the expression in that article contains a typographical error. This error does not affect any results presented in that work (D. Vech 2019, personal communication).
}

shown as a green dashed line in Figure 1(b). We reject any intervals where $n_{s 2}>1.7$ to ensure we resolve the dissipation scale spectra. We emphasize that the described procedure is not compromised by removing the $1 \mathrm{~Hz}$ peak as $f_{b}$ values, not plotted here, remain in the interval $0.2-0.4 \mathrm{~Hz}$ throughout the entire mission, as was elaborated on in detail by Bourouaine et al. (2012).

Given a value for $\delta B$ extracted from $P_{B}(f)$ (Equation (8)), we calculate $\epsilon$ and $Q_{\perp}$ using Equations (1) and (2). From a total of 259,80110 minute intervals, we have 82,881 with $\Delta t \leqslant 0.5 \mathrm{~s}$, 24,615 of which satisfy the above described criteria. Out of these, 14,954 intervals with $\beta_{\|}<0.3$ are used in this study as Equation (2) is derived in the low- $\beta$ limit (see Section 1). In Figure 1(a) we show the number of intervals used as a function of radial distance, using 20 linear bins 0.035 au wide. Notably, the percentage of usable measurements significantly decreases for $r>0.6 \mathrm{au}$. This is due to instrument related issues, as described in the following section.

\section{Properties and Limitations of the E2 Magnetometer}

As the Helios instrument documentation does not provide a noise level for E2 magnetometers ${ }^{8}$ it was necessary to extract it from the data. The available E2 data set provides measurements with accuracy of $0.1 \mathrm{nT}$ for each of the magnetic field components. To check for a possible noise floor, we produced histograms of the radial component on Figure 2, with bin sizes of $0.01 \mathrm{nT}$ (a) and $0.1 \mathrm{nT}$ (b), using a total of $1.7 \times 10^{8}$ measurements with no averaging over time intervals. It is notable from panel (a) that there is no concentration point at $B_{r}=0$. As the instrument sensitivity, extracted from the data, is $B_{s}=0.1 \mathrm{nT}$ (the radial component can only have values $B_{r}=n B_{s}$, where $n$ is an integer), we conclude that the E2 noise level is less or equal to this value. The sensitivity $B_{s}$ has major influence on the data processing, as it sets the lowest possible level of magnetic field PSD, which is independent of

\footnotetext{
8 The exact determination of the E2 noise level is an ongoing task of the Helios data archive team (Salem 2017).
} 


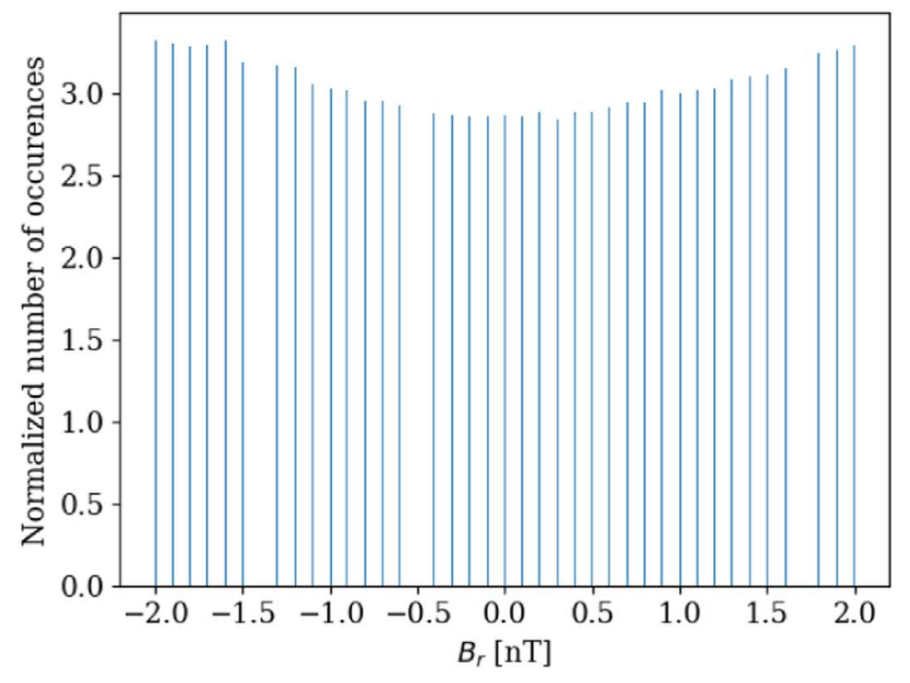

(a)

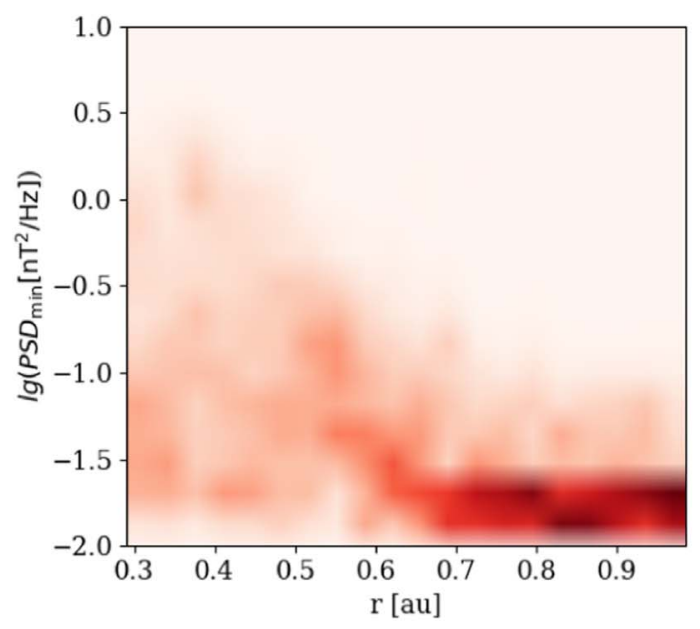

(c)

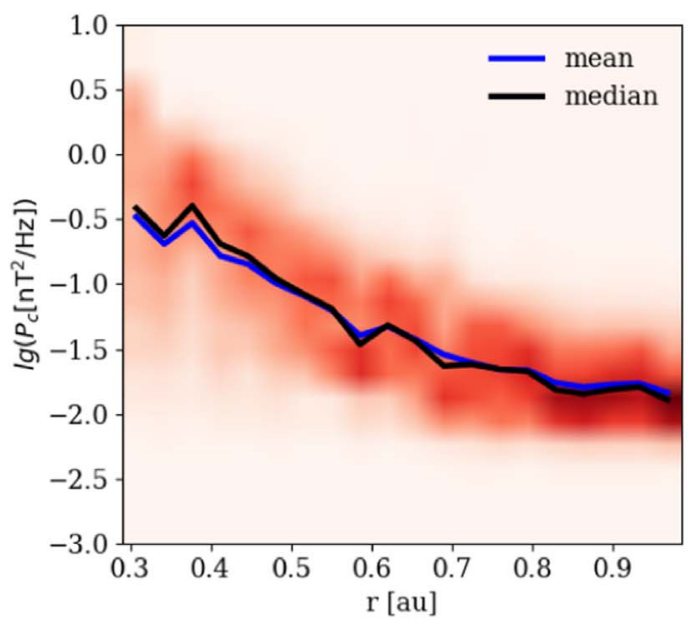

(e)

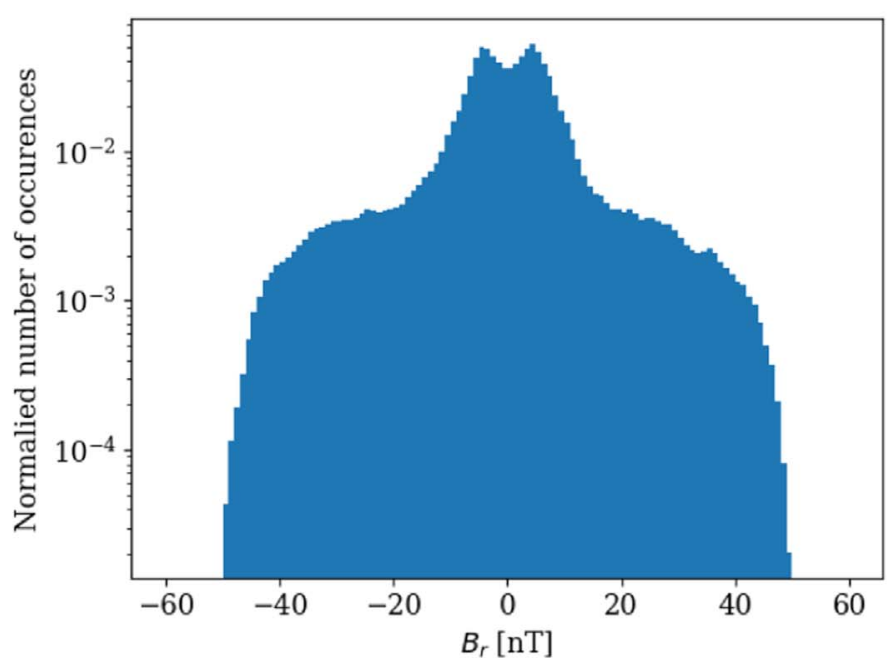

(b)

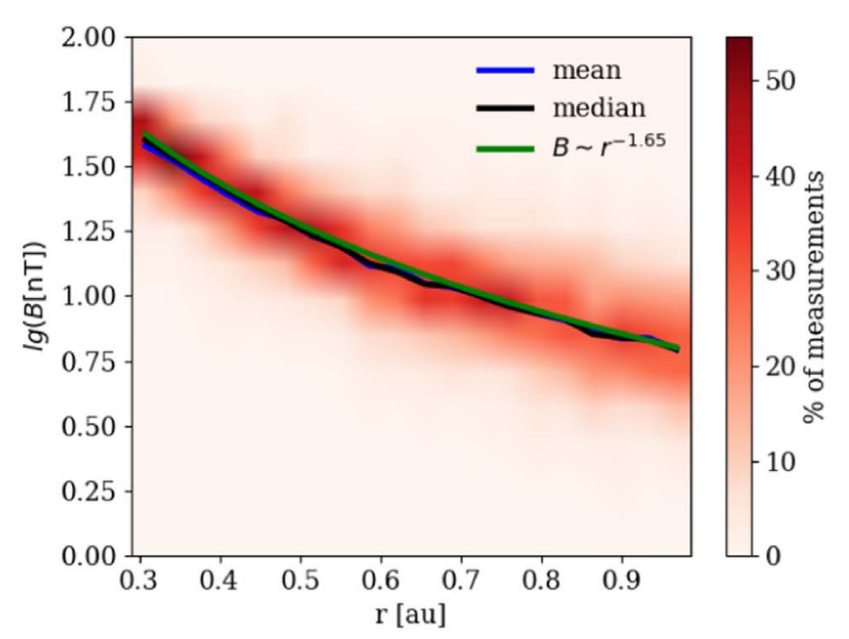

(d)

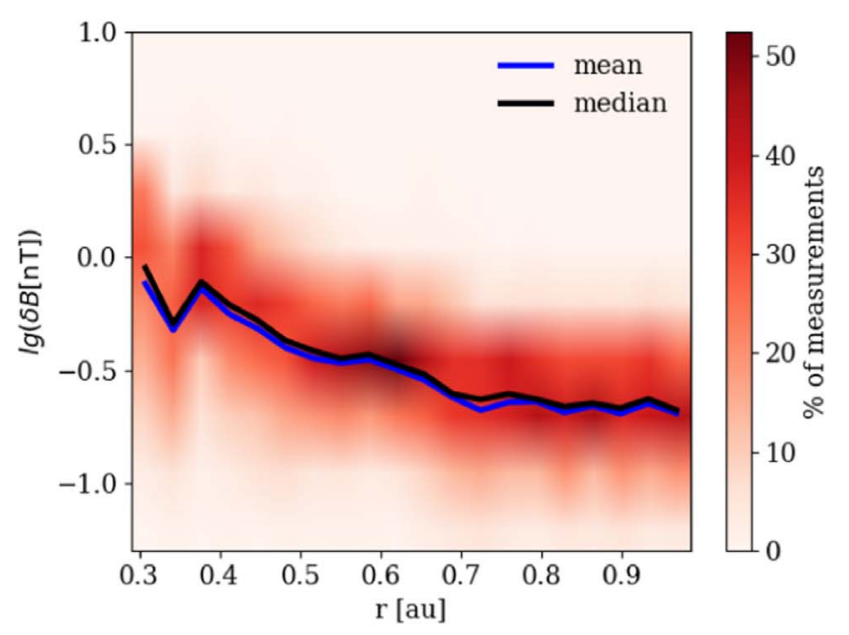

(f)

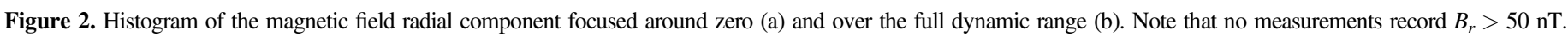

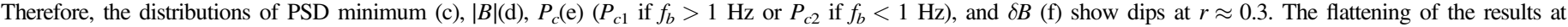
$r>0.65$ au is due to limited E2 sensitivity. Here, $\lg$ stands for the base 10 logarithm. 
frequency, to $\mathrm{B}_{s}^{2}$ (panel (c)). Histograms for the other two components, not shown, behave in a similar fashion. Another important point is that no measurements record $B_{r}>50 \mathrm{nT}$. In the instrument team reports, it is noted that saturation might occur at this level, which is confirmed in panel (d).

These instrumental limitations have important consequences on the reliability of the measurements. From panel (d), we see the magnetic field value trend of $B \sim r^{-1.65}$ that has previously been reported beyond $r \approx 0.35$ au (see, e.g., Hellinger et al. 2013). Below this distance, the magnetometer saturates and any increase in the measured field intensity comes only from tangential and normal components. This has a major influence on derived values of $\delta B$ and, consequently, $\delta v$ and $\epsilon$, leading to significant underestimation, as shown in panel (f).

On the other hand, at radial distances $r>0.65 \mathrm{au}$, it is notable that, even though measured magnetic field values follow the expected radial trend, $\delta B$ results reach a constant value due to the E2 sensitivity floor. In order to verify this statement, we analyzed the minimum of the power spectra, as well as behavior of the $1 \mathrm{~Hz}$ cutoff data, panels (c) and (e), respectively, where a floor starting at $r \approx 0.65$ au as well as a local minimum at $r \approx 0.3$ au are visible. We have much less confidence in results that come from these two regions, and consider them separately from the region between 0.35 and 0.65 au. Note that the percentage of intervals fitting our criteria significantly decreases for larger radial distances (Figure 1(a)) even though the total number of results remains similar due to the spacecraft spending more time in this region.

\section{Results of Helios Statistical Study}

The results of our statistical study are shown in Figure 3. Mean values of $\epsilon$, shown on panel (a), are constant throughout the range of $r=0.4-0.65 \mathrm{au}$, where the Helios magnetometer observations are reliable. Using the derived $\epsilon$, we calculate $Q_{\perp}$ (panel (b)), using $c_{1}=0.75$ and $c_{2}=0.34$, values extracted from the test-particle simulations by Chandran et al. (2010). As discussed in Section 5, while the value of these parameters can have significant influence on the amplitude of $Q_{\perp}$, they are expected to remain constant as a function of radial distance, and thus will not affect radial trends in $Q_{\perp}$.

The average value of $Q_{\perp}$ decreases with radial distance (red line), and can be logarithmically fit as $Q_{\perp} \sim r^{-2.5 \pm 0.4}$. We compare our results with two previous models of solar wind heating. A simple model used by Bourouaine \& Chandran (2013; see Equation (6) of that article), when applied to our data set, reports a weaker radial trend of $Q_{\perp} \sim r^{-2.08}$ (black dashed line on panel (c)). On the other hand, a two-fluid theoretical model by Chandran et al. (2011) that includes the effects of SH, as well as proton and electron Landau damping, reports $Q_{\perp} \sim r^{-2.9}$ as the perpendicular heating rate (green dashed line). It also predicts that the relative importance of $\mathrm{SH}$ compared to the electron heating should decrease beyond $r>0.3$ au. We note that the authors of this model state that its primary purpose was to predict conditions closer to the Sun, and that it may become increasingly inaccurate for larger radial distances due to violation of assumptions on the energy cascade times and relation between Elsassër variables.

The average measured amplitude of the heating, for $c_{1}=0.75$ and $c_{2}=0.34$ and considering only radial distances with reliable measurements (green dots on Figure 3(c)), is found to be $\sim 10^{3} \mathrm{~W} \mathrm{~kg}^{-1}$ at $1 \mathrm{au}$, increasing up to $4.8 \times 10^{4} \mathrm{~W} \mathrm{~kg}^{-1}$ at $0.15 \mathrm{au}$, with the radial trend described above. Comparing it to the results from Chandran et al. (2011) and Bourouaine \& Chandran (2013), we note that the contribution of SH from low $\beta$ solar wind streams is an order of magnitude below the total heating values predicted by these authors, increasing to $20 \%-40 \%$ at 0.15 au. However, these contributions are strongly dependent on the choice of the model constants (see Section 5).

In order to compare our results with available models obtained from observations, we use empirical total heating rates $Q(r)$ derived by Hellinger et al. (2011) for $v_{\mathrm{sw}}>600 \mathrm{~km} \mathrm{~s}^{-1}$ and Hellinger et al. (2013) for $v_{\mathrm{sw}}<400 \mathrm{~km} \mathrm{~s}^{-1}$. We recalculate $\epsilon$ and $Q_{\perp}$ using only intervals that meet these bulk velocity criteria. For the slow wind, where 4325 out of 14954 intervals are used, the estimated SH rate is decreased compared to the total empirical heating rate $\left(Q_{\perp} \sim r^{-0.6 \pm 0.4}\right.$ against $Q \sim r^{-1.2}$ ), as shown in panel (e). However, results for the fast solar wind, using 4225 out of 14954 intervals, are fundamentally different. Average values of $\epsilon$ are systematically higher in this case (panel (d)), which increases estimated $Q_{\perp}$ for the fast wind by an order of magnitude, as shown in panel (f), demonstrating that the majority of total heating, as well as the contribution from $\mathrm{SH}$, happens in the fast solar wind streams. This amplitude increase is in agreement with the empirical model, but the radial trend of our derived SH rate in the fast wind is much steeper, $Q_{\perp} \sim r^{-3.1 \pm 0.5}$ compared to the empirical $Q \sim r^{-1.8}$. Therefore, the increasingly important role of SH at closer radial distances to the Sun is driven by intervals of fast solar wind. Additionally, since the Helios aphelion (with operational instruments) is at $r \approx 0.98 \mathrm{au}$, this distance is the limit of the observational models compared with our data. The heating rates extracted here also compare well with the results of MacBride et al. (2008), who followed the formalism developed by Politano \& Pouquet (1998a, 1998b) to account for the total dissipation rate of the turbulent energy in the inertial range. By studying $7 \mathrm{yr}$ of $A C E$ data at $\mathrm{L} 1$, they found similar dissipation rates to those theoretically predicted (Chandran et al. 2011; Bourouaine \& Chandran 2013) and extrapolated from observations at smaller radial distances (Hellinger et al. 2011, 2013).

Another major feature of our results is the spread of $Q_{\perp}$ values throughout 10 orders of magnitude. The shape of $1 \mathrm{D}$ histograms of $\lg \left(Q_{\perp}\right)$ (not shown) obeys Gaussian distribution with low power tails, while $Q_{\perp}$ distributions are highly skewed with large differences between mean and median, as shown in Figure 3(b), with only $15 \%$ of the measured intervals having amplitudes larger than the average value. These high values determine the bulk SH characteristics and will be separately discussed in the following section.

\section{Discussion}

Following the method and results presented in Sections 2 and 4 there are several features and limitations that demand further discussion. First, we only consider low $\beta$ plasma streams, which are, on average, expected to contribute less to $Q_{\perp}$ than cases with higher $\beta$, according to recent theoretical results (Hoppock et al. 2019). The effect of SH in plasma with $\beta \gtrsim 1$ will be investigated in future work (M. M. Martinović et al. 2019, in preparation).

Variations in the three-dimensionless parameters used in this model, $\sigma, c_{1}$, and $c_{2}$, may induce a systematic error in our results. We assumed that only Alfvénic fluctuations are present, and therefore asserted a relation between magnetic and velocity 


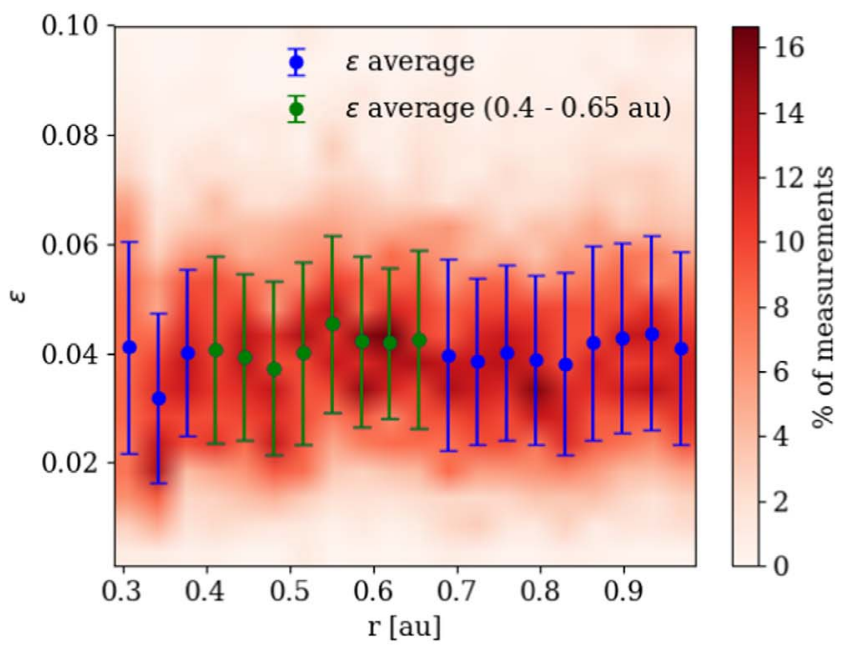

(a)

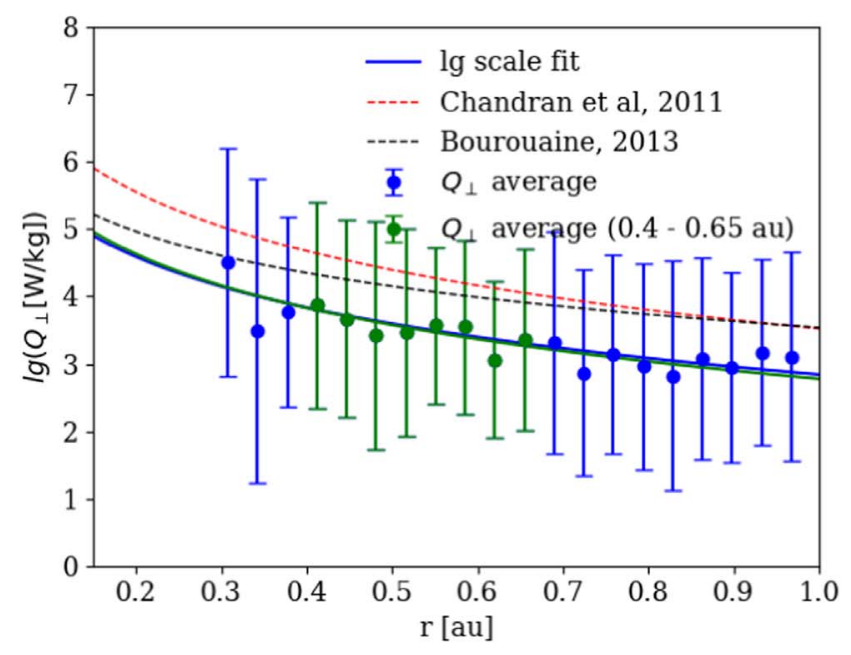

(c)

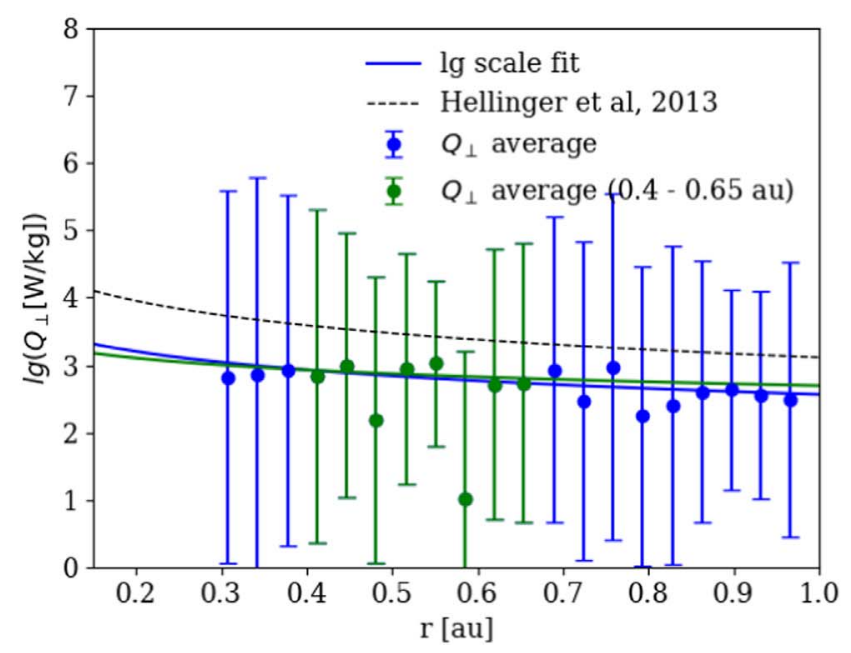

(e)

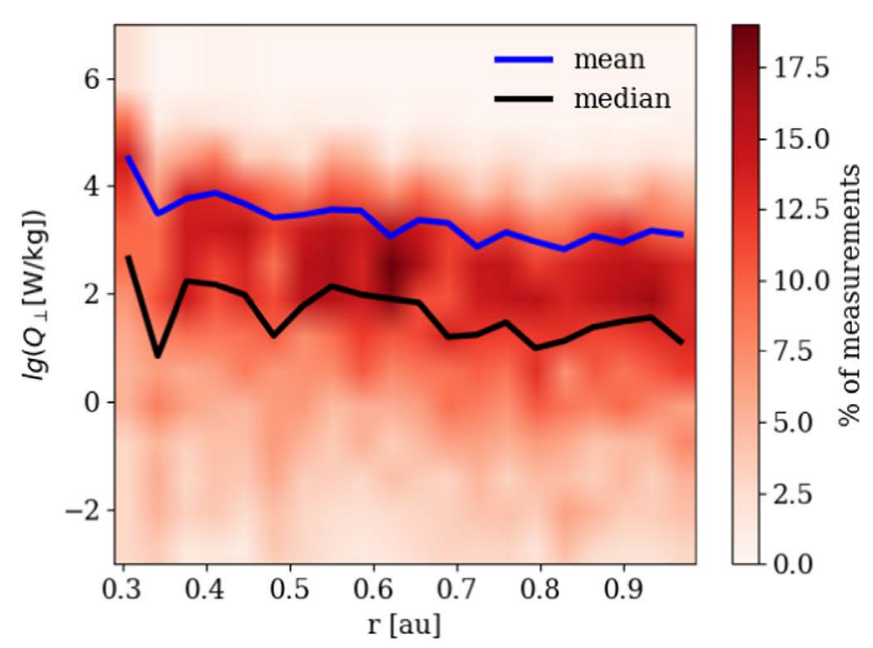

(b)

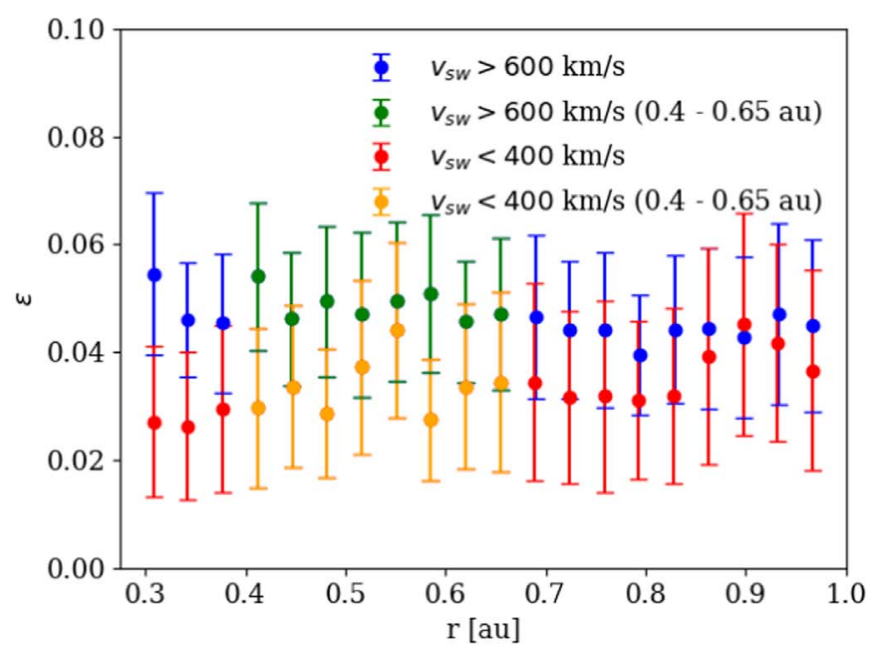

(d)

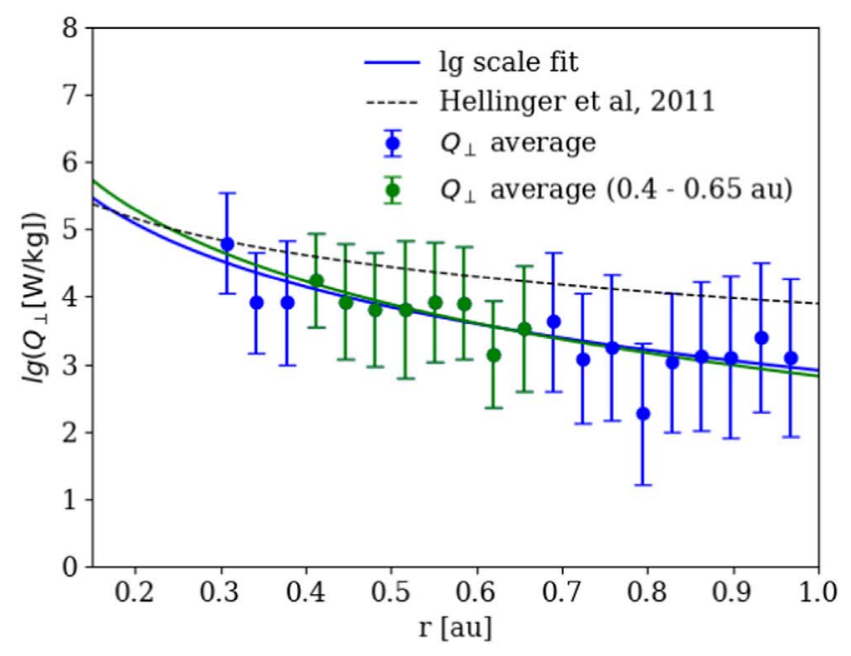

(f)

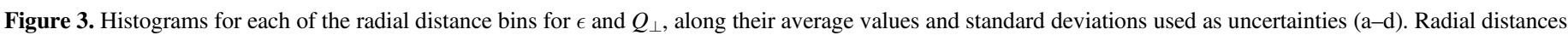

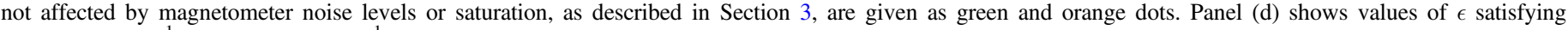
$v_{\mathrm{sw}}<400 \mathrm{~km} \mathrm{~s}^{-1}$ or $v_{\mathrm{sw}}>600 \mathrm{~km} \mathrm{~s}^{-1}$, and the associated $Q_{\perp}$ compared with their respective empirical models are given in panels (e) and (f). 


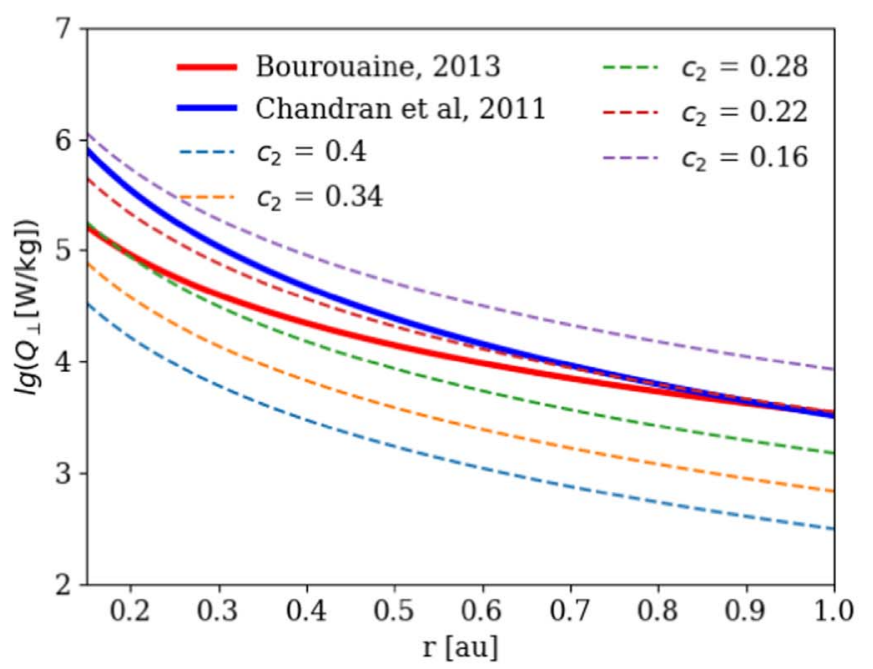

(a)

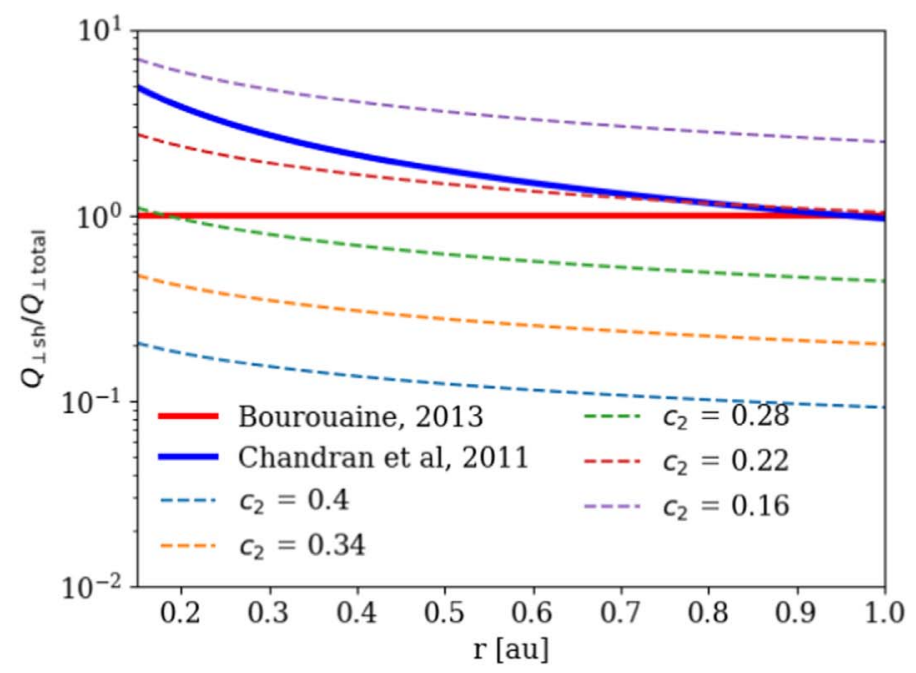

(b)

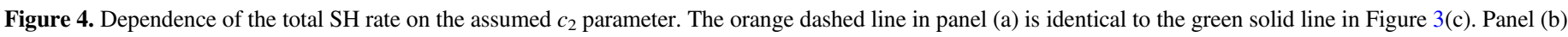
illustrates the ratio of $Q_{\perp \mathrm{SH}}$ and $Q_{\perp \text { total }}$ from Bourouaine \& Chandran (2013) and Chandran et al. (2011).

fluctuations in Equation (6), setting a constant value of $\sigma=1.19$. This parameter is expected to be lower for the case of non-Alfvénic fluctuations (see, e.g., Barnes \& Hollweg 1974; Roberts et al. 1987), potentially becoming less than unity. However, by comparing panels (e) and (f) on Figure 3, it is notable that the highest dissipation rates are observed in the fast solar wind, where protons exhibit strong temperature anisotropy and highly Alfvénic properties (Stansby et al. 2019). We therefore argue that usage of the constant $\sigma$ is still justified for obtaining average $Q_{\perp}$. This enables us to predict that $\mathrm{SH}$ has an increasingly important role in the total solar wind heating rate at $r<0.3 \mathrm{au}$, even though combined results shown on panel (c), along with very large uncertainties, indicate that its relative importance remains approximately the same for $r=$ 0.35-0.65 au. Additional concern arises from some recent hybrid MHD simulation results (Franci et al. 2015), showing that magnetic and velocity fluctuations tend to decouple at the proton inertial length. This issue will be investigated through variations of $\sigma$ in future work.

The values of $c_{1}=0.75$ and $c_{2}=0.34$ are chosen using results from test-particle simulations. Values for these parameters have also been extracted from RMHD simulations (Xia et al. 2013), with results summarized in Table 1 of that article and in Figure 5 of Bourouaine \& Chandran (2013). These works argue that realistic values for $c_{2}$ can be as low as 0.15 , similar to the value of $c_{2}=0.17$ assumed by Chandran et al. (2011). We examine this range of values below, with the understanding that additional refinements to values of $c_{1}$ and $c_{2}$ may become available from more realistic Vlasov simulations.

As $c_{2}$ participates in the exponential term in Equation (2), its variation has the largest impact on $Q_{\perp}$. We examine its effect on $Q_{\perp}$ by recalculating Figure 3(c) using $c_{2}=0.16-0.4$ separated by increments of 0.06 ; this range covers parameter values considered by previous authors. As shown in Figure 4, varying $c_{2}$ by a factor of 2.5 increases $Q_{\perp}$ by one and a half orders of magnitude. We note that as $\epsilon$ linearly scales with $\sigma$, variations of $c_{2}$ can be used to examine variations in $\sigma$. Testparticle simulations by Chandran et al. (2010) have demonstrated that $c_{1}$ and $c_{2}$ are fairly insensitive to plasma parameters (see Figure 4 of that article) so we do not expect significant radial variation of those parameters. Note that for the case of $c_{2}=0.22, Q_{\perp}$ is consistent with the heating rate from the Bourouaine \& Chandran (2013) model at large radial distances, but then exceeds the model by almost a factor of 2 at smaller distances. Therefore, if $Q_{\perp}$ is not to exceed $Q_{\text {total }}$ at small radial distances, it is expected to contribute relatively less and less at larger radial distances for values of $c_{2}>0.22$. On the other hand, comparison with the Chandran et al. (2011) model, which includes both proton heating due to Landau damping and $\mathrm{SH}$ and electron heating, has a steeper radial dependence than either the Bourouaine \& Chandran (2013) model or our observations, matching our model for $c_{2}=0.22$ but exceeding it by a factor of 3 at $r=0.2 \mathrm{au}$. Comparison with this model states that values $c_{2}<0.22$, with radial trends calculated in Section 4 , can be realistic within the range of uncertainties of our results, shown on Figure 3(c).

Even though it was noted above that the results for $r<0.35$ au are not reliable due to saturation in magnetic field measurements, we will still comment on the measured $\epsilon$ values for the radial bin $r<0.3 \mathrm{au}$. The E2 saturation is expected to cause an underestimation of the measured $\delta B, \delta v$, and $\epsilon$, as seen in the bin at $r \sim 0.3$. However, the measured $\epsilon$ is one of the largest in Figure 3(a). This hints that SH might be the dominant process close to the Sun, and potentially the process active in the zone of preferential ion heating predicted by Kasper et al. (2017) to extend tens of solar radii from the Sun's surface. Given the instrumental limitations described earlier in this work, this conclusion should be considered tentative and must be verified by observations of future missions. Parker Solar Probe (Fox et al. 2016) will offer the first opportunity to provide such verification.

A last effect should be examined in future work. The applicability of SH in highly intermittent turbulence, where the fluctuating fields are larger than their rms values, potentially leading to increased heating (Dmitruk et al. 2004; Chandran et al. 2010), is an open question. Recent results (Mallet et al. 2019) indicate that the effects of intermittency can be incorporated into models for nonlinear heating rates and play an important role in enhancing the solar wind heating due to nonlinear mechanisms such as $\mathrm{SH}$. This model will be 
confronted with solar wind observations, and is the focus of future work.

M.M.M. was financially supported by the Ministry of Education, Science and Technological Development of Republic of Serbia through financing the project ON176002. K.G.K was supported by NASA grant NNX16AM23G. The authors would like to thank Chadi Salem and Daniel Vech for clarifying conversations. The data used for this project were sourced from NASA CDAweb (footnote 5) and the SSL Helios repository (footnote 6).

\section{ORCID iDs}

Mihailo M. Martinović (D) https://orcid.org/0000-00027365-0472

Kristopher G. Klein (iD https://orcid.org/0000-0001-6038-1923 Sofiane Bourouaine (ib https://orcid.org/0000-0002-2358-6628

\section{References}

Bale, S. D., Kellogg, P. J., Mozer, F. S., Horbury, T. S., \& Reme, H. 2005 PhRvL, 94, 215002

Barnes, A., \& Hollweg, J. V. 1974, JGR, 79, 2302

Belcher, J. W., \& Davis, L., Jr. 1971, JGR, 76, 3534

Belcher, J. W., Davis, L., Jr., \& Smith, E. J. 1969, JGR, 74, 2302

Bourouaine, S., Alexandrova, O., Marsch, E., \& Maksimovic, M. 2012, ApJ, 749, 102

Bourouaine, S., \& Chandran, B. D. G. 2013, ApJ, 774, 96

Chandran, B. D. G., Dennis, T. J., Quataert, E., \& Bale, S. D. 2011, ApJ, 743, 197

Chandran, B. D. G., Li, B., Rogers, B. N., Quataert, E., \& Germaschewski, K. 2010, ApJ, 720, 503

Chen, C. H. K., Horbury, T. S., Schekochihin, A. A., et al. 2010, PhRvL, 104, 255002

Chen, C. H. K., Klein, K. G., \& Howes, G. G. 2019, NatCo, 10, 740

Cho, J., \& Lazarian, A. 2004, ApJL, 615, L41

Coleman, P. J., Jr. 1968, ApJ, 153, 371

Dmitruk, P., Matthaeus, W. H., Milano, L. J., et al. 2002, ApJ, 575, 571

Dmitruk, P., Matthaeus, W. H., \& Seenu, N. 2004, ApJ, 617, 667

Drake, J. F., Cassak, P. A., Shay, M. A., Swisdak, M., \& Quataert, E. 2009, ApJL, 700, L16

Drake, J. F., \& Swisdak, M. 2012, SSRv, 172, 227

Echim, M. M., Lemaire, J., \& Lie-Svendsen, y. 2011, SGeo, 32, 1

Fox, N. J., Velli, M. C., Bale, S. D., et al. 2016, SSRv, 204, 7

Franci, L., Landi, S., Matteini, L., Verdini, A., \& Hellinger, P. 2015, ApJ, 812,21

Goldreich, P., \& Sridhar, S. 1995, ApJ, 438, 763
Hellinger, P., Matteini, L., Štverák, Š., Trávníček, P. M., \& Marsch, E. 2011, JGRA, 116, A09105

Hellinger, P., TráVníček, P. M., Štverák, Š., Matteini, L., \& Velli, M. 2013, JGRA, 118, 1351

Hollweg, J. V. 1973, JGR, 78, 3643

Hollweg, J. V. 1999, JGR, 104, 14811

Hollweg, J. V., \& Isenberg, P. A. 2002, JGRA, 107, 1147

Hoppock, I. W., Chandran, B. D. G., Klein, K. G., Mallet, A., \& Verscharen, D. 2019, JPIPh, 84, 905840615

Howes, G. G., Cowley, S. C., Dorland, W., et al. 2008a, JGRA, 113, A05103

Howes, G. G., Dorland, W., Cowley, S. C., et al. 2008b, PhRvL, 100, 065004

Howes, G. G., Klein, K. G., \& Li, T. C. 2017, JPIPh, 83, 705830102

Kasper, J. C., Klein, K. G., Weber, T., et al. 2017, ApJ, 849, 126

Kasper, J. C., Maruca, B. A., Stevens, M. L., \& Zaslavsky, A. 2013, PhRvL, 110,091102

Klein, K. G., \& Howes, G. G. 2016, ApJL, 826, L30

Klein, K. G., Howes, G. G., \& Tenbarge, J. M. 2017, JPIPh, 83, 535830401

Lehe, R., Parrish, I. J., \& Quataert, E. 2009, ApJ, 707, 404

MacBride, B. T., Smith, C. W., \& Forman, M. A. 2008, ApJ, 679, 1644

Mallet, A., Klein, K. G., Chandran, B. D. G., et al. 2019, JPIPh, submitted

Mallet, A., Schekochihin, A. A., \& Chandran, B. D. G. 2015, MNRAS, 449, L77

Markovskii, S. A., Vasquez, B. J., \& Chandran, B. D. G. 2010, ApJ, 709, 1003

Marsch, E., Schwenn, R., Rosenbauer, H., et al. 1982, JGR, 87, 52

Matthaeus, W. H., Zank, G. P., Oughton, S., Mullan, D. J., \& Dmitruk, P. 1999, ApJL, 523, L93

Matthias, A. R., Lazarus, A. J., \& Steinberg, J. T. 2001, GeoRL, 136, 2767

Musmann, G., Neubauer, F. M., Maier, A., \& Lammers, E. 1975, RF, 19, 232

Osman, K. T., Matthaeus, W. H., Hnat, B., \& Chapman, S. C. 2012, PhRvL, 108,261103

Parker, E. N. 1958, ApJ, 128, 664

Politano, H., \& Pouquet, A. 1998a, GeoRL, 25, 273

Politano, H., \& Pouquet, A. 1998b, PhRvE, 57, R21

Porsche, H. 1981a, ESA Spec. Publ., 164, 43

Porsche, H. 1981b, DFVLR, 33, 33

Quataert, E. 1998, ApJ, 500, 978

Roberts, D. A., Klein, L. W., Goldstein, M. L., \& Matthaeus, W. H. 1987, JGR, 92, 11021

Salem, C. 2017, Helios 1 and 2 In Situ Data Archive: Project Action Item List, (Berkeley, CA: Univ. California, Berkeley), http://helios-data.ssl.berkeley. edu/data/ActionItemList_v2.pdf

Schekochihin, A. A., Cowley, S. C., Dorland, W., et al. 2009, ApJS, 182, 310

Schwenn, R., Rosenbauer, H., \& Miggenrieder, H. 1975, RF, 19, 226

Stansby, D., Horbury, T. S., \& Matteini, L. 2019, MNRAS, 482, 1706

Stansby, D., Salem, C., Matteini, L., \& Horbury, T. 2018, SoPh, 293, 155

Sturrock, P. A., \& Hartle, R. E. 1966, PhRvL, 14, 628

Vech, D., Klein, K. G., \& Kasper, J. C. 2017, ApJL, 850, L11

Vech, D., Mallet, A., Klein, K. G., \& Kasper, J. C. 2018, ApJL, 855, L27

Velli, M., Grappin, R., \& Mangeney, A. 1989, PhRvL, 63, 1807

Villante, U. 1980, JGR, 85, 6869

Wolff, C. L., Brandt, J. C., \& Southwick, R. G. 1971, ApJ, 165, 181

Xia, Q., Perez, J. C., Chandran, B. D. G., \& Quataert, E. 2013, ApJ, 776, 90 